\title{
Atherosclerosis and Sudden Death and Their Relationship to Visceral Fat in Men Under 40 With no History of Cardiovascular Diseases and Normal BMI
}

\author{
Farrokh Taftachi, ${ }^{1}$ Leyla Abdolkarimi, ${ }^{1,}$ Maryam Ameri, ${ }^{2}$ Azade Memarian, ${ }^{2}$ Zeynalabedin Malekpoor, ${ }^{3}$
} and Mostafa Jafarzadeh ${ }^{4}$

${ }^{1}$ Rajaie Cardiovascular, Medical and Research Center, Iran University of Medical Sciences, Tehran, IR Iran ${ }^{2}$ Department of Legal Medicine, Iran University of Medical Sciences, Tehran, IR Iran

${ }^{3}$ Forensic Specialist, Shahrekord University of Medical Sciences, Kashani Hospital, Shahrekord, IR Iran

${ }^{4}$ Forensic Specialist, Legal Medicine Research Center, Legal Medicine Organization, Tehran, IR Iran

"Corresponding author: Leyla Abdolkarimi, Rajaie Cardiovascular, Medical and Research Center, Iran University of Medical Sciences, Tehran, IR Iran. Tel: +98-2123912005, E-mail: leyly.abdolkarimi@yahoo.com

Received 2016 August 14; Revised 2016 September 07; Accepted 2016 September 07.

\begin{abstract}
In addition to abdominal fat thickness, the question arises whether other visceral fat deposits, including epicardial and mesenteric fat, even in men with a normal body mass index (BMI < 30 and acceptable waist/hip ratio [WHR]) can lead to atherosclerosis and cardiac hypertrophy. The present study was conducted to compare the distribution of visceral fat between men under 40 who experienced sudden death due to coronary artery obstruction and a control group who have passed from traffic accidents in Tehran. Autopsy samples were collected from a total of 94 men and divided into 2 groups of 47 men. The case group (sudden death from coronary obstruction) and the control group (death due to traffic accidents) were matched in terms of age, height and weight. BMI and waist/hip ratio were within the acceptable range in both groups. Neither of the groups had cardiovascular or other diseases. The findings of the study showed a greater thickness of subcutaneous abdominal fat around the navel and a higher epicardial fat, mesenteric weight, heart mass and heart mass to height ratio in the group of men who died due to coronary artery obstruction. Accordingly, even in seemingly healthy men under 40 with no obese men (with normal BMI and acceptable WHR), the accumulation of internal visceral fat such as subcutaneous abdominal fat around the navel and epicardial and mesenteric fat can contribute to coronary artery obstruction and cardiac hypertrophy.
\end{abstract}

Keywords: Atherosclerosis, BMI, Cardiac Hypertrophy, Coronary Artery Obstruction, Epicardial Fat, Obesity, WHR, Visceral Fat

\section{Background}

BMI (body mass index) does not demonstrate the distribution of fat in the body, and central fat is mostly associated with cardiovascular diseases $(1,2)$. In fact, cardiovascular events are predominantly associated with the accumulation of abdominal fat (3). Angiographic results have also confirmed the relationship between coronary stenosis and abdominal obesity (4). Even in men under 40 with no history of cardiac diseases and with a normal body mass in$\operatorname{dex}(\mathrm{BMI}<30)$, abdominal fat is still associated with coronary artery lesions (5). Since the accurate measurement of the thickness of abdominal fat around the navel is difficult the waist/hip ratio (WHR) is used as a proper measure of abdominal obesity (6). WHR is linked to coronary artery diseases according to several studies $(1,6)$ and even individuals with normal weight $(\mathrm{BMI}<30)$ but large waistlines are also at risk for the development of cardiovascular diseases (7).

Whether people with acceptable WHR who are prone to the risk of atherosclerosis and sudden death? In recent years when working in autopsy salons in the Tehran Lagal medicine center, we had a number cases of sudden deaths due to atherosclerosis in men under 40 with no history of cardiovascular diseases, normal BMI $<30$ and acceptable WHR. In previous papers the role of visceral fat has been noted in such cases (5, 8-10). The question arises whether visceral fat deposits, including epicardial and mesenteric fat, even in men with normal body mass indexes, will lead to atherosclerosis and cardiac hypertrophy.

Epicardial fat covers $80 \%$ of the heart surface but accounts for only $20 \%$ of its mass. Autopsy studies have reported a mean percentage of epicardial fat to heart mass ratio of $15.2 \%$ to $25.2 \%$ in men and $19.5 \%$ to $21.7 \%$ in women.

Copyright (c) 2016, Rajaie Cardiovascular Medical and Research Center, Iran University of Medical Sciences. This is an open-access article distributed under the terms of the Creative Commons Attribution-NonCommercial 4.0 International License (http://creativecommons.org/licenses/by-nc/4.0/) which permits copy and redistribute the material just in noncommercial usages, provided the original work is properly cited. 
There is a direct relationship between epicardial fat and abdominal fat (11). In addition to abdominal fat, studies have also proposed the possible effect of epicardial fat on atherosclerosis (3). Moreover, the thickness of epicardial fat is a predictor of the volume of visceral fat irrespective of BMI (12). For instance, epicardial fat can affect atherogenesis and myocardial function, and those parts of the coronary artery that have no epicardial fat or are farther away from these fat deposits are at a lower risk of developing atheromatous plaques (13).

\section{Objectives}

The present study was conducted on a population of Iranian men under 40 to investigate the role of visceral fat (abdominal fat around the navel, epicardial fat, mesenteric, and omental fat) in coronary artery pathology via autopsy.

\section{Methods}

The study enrolled all men under 40 who experienced sudden death and were referred to Tehran Coroner's office, where their autopsies had shown coronary artery obstruction as the cause of their death. Additionally, from amongst the above-mentioned, only those with the BMI < 30 and with acceptable WHR) < 0.9) and who had no history of cardiovascular diseases, dyslipidemia, alcohol or drug use, physical disability and other diseases were included. A total of 50 samples were collected over a period of about 1 year, from May 2013 to March 2014. Histopathological tests were carried out and 3 cases of myocarditis were excluded, thus the case group consisted of 47 people.

The control group were men who died from traffic accidents in Tehran. For the control group, the autopsy samples of 47 deceased men under 40 who had died from traffic accidents with no history of cardiovascular diseases, hypertension, dyslipidemia, alcohol or drug use, physical disability and other diseases and with an acceptable BMI (< $30)$ and WHR $(<0.9)$ were selected. Given the abundance of bodies brought in for traffic accidents, the control group was selected consecutively using the inclusion data.

The bodies were all naked on the autopsy tabulation. Then height, weight, waist and hip circumferences were measured. After the autopsy, omentum, mesentery and heart from the base were removed and washed. Fat thickness around the navel, heart, omentum and mesentery weight was measured. Epicardial fat was carefully removed and weighed. It also with rutin coronary cuts the amount of atherosclerosis in the coronary arteries was determined.

\subsection{Statistical Analysis}

Data was analyzed by SPSS16 for Windows (SPSS Inc., Chicago, Illinois). The comparison between two groups was performed using chi-square for categorical and Student's t-test for interval data. $\mathrm{P}<0.05$ is considered as statistically significant.

\section{Results}

Table 1 shows that the case and control groups were not significantly different in age, BMI and WHR. BMI and WHR were within the acceptable range in both groups.

Before comparing the case and control group in terms of the distribution of visceral fat, we first determined the level of coronary obstruction in the two groups by cardiac autopsy and coronary artery examination. Table 2 presents the results obtained from the investigations. This table displays the percentage of blockage in the coronary arteries and show coronary arteries obstruction was significantly lower in the control group than in the case group.

Table 3 reveals higher thickness of subcutaneous abdominal fat around the navel, epicardial fat mass and mesenteric weight in the cases than in the controls. However, no differences were observed between the 2 groups with respect to omental weight. Moreover, heart mass and heart mass to height ratio $(\mathrm{g} / \mathrm{cm} \times 2 / 7)$ was higher in the cases than in the controls, which is due to both higher epicardial fat and cardiac hypertrophy.

\section{Discussion}

In a study conducted in 1996 at Oulu University in Finland, Kortelaine took autopsy samples from 30 men who had no history of cardiovascular diseases and who had experienced sudden cardiac death. He measured their height, weight, BMI, abdominal fat thickness, WHR, heart mass and epicardial, omental, and mesenteric fat and demonstrated a relationship between visceral fat distribution and coronary artery pathology (9). These findings chime in with the results of some other studies $(5,8,10)$.

The findings of the current study revealed higher subcutaneous abdominal fat around the navel, epicardial fat, mesenteric weight, heart mass and heart mass to height ratio $(\mathrm{g} / \mathrm{cm} \times 2 / 7)$ in the group that had experienced sudden death due to coronary obstruction than in the group that had died in traffic accidents.

Our results also showed that in seemingly healthy men under 40 with normal BMI and WHR, measuring the internal visceral fat, including subcutaneous abdominal fat around the navel, epicardial fat and mesenteric weight, can help determine the likelihood of coronary obstruction 
Table 1. Compration of Background Data Between Case and Control Groups

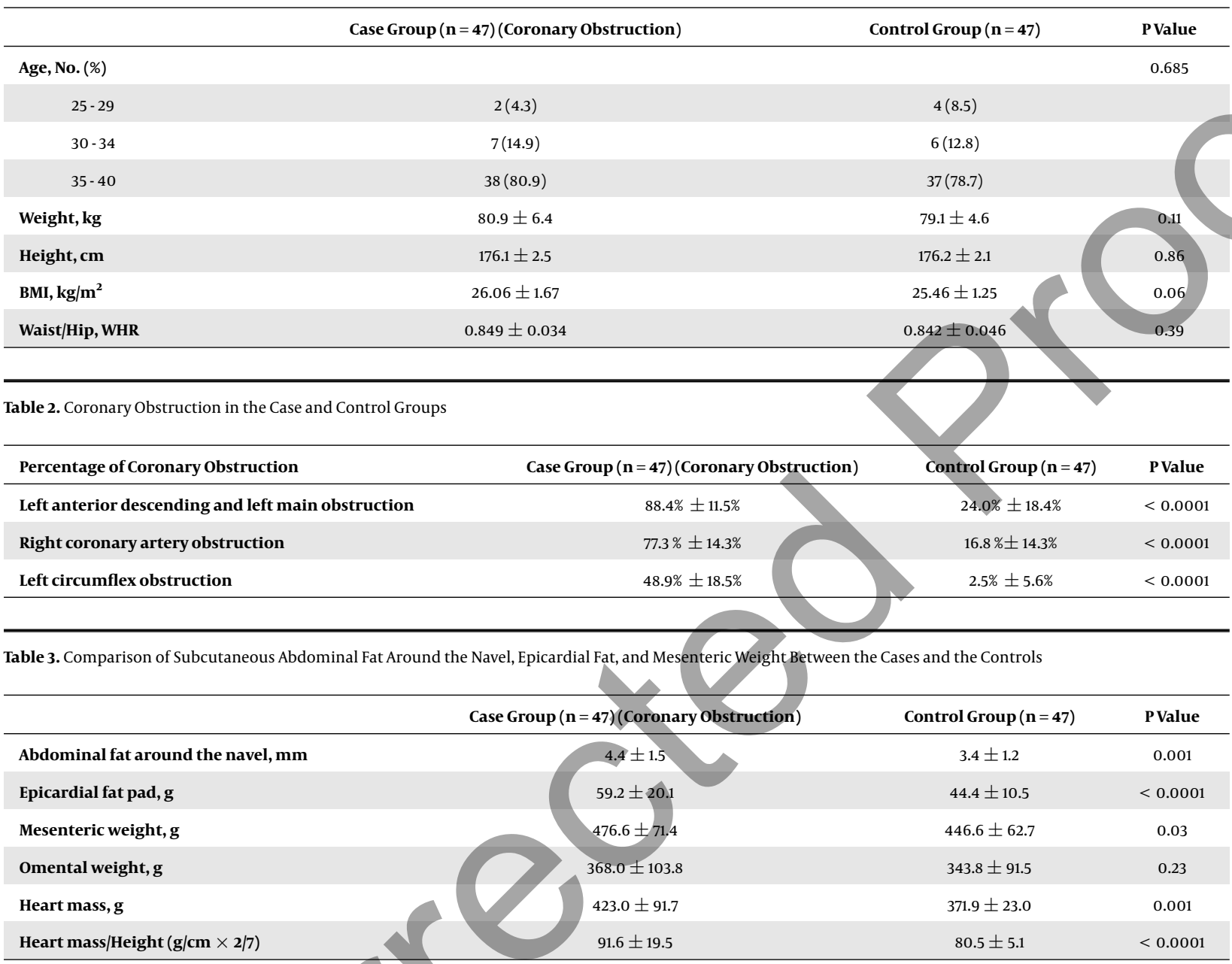

and cardiovascular diseases. As a result, in addition to apparent obesity and increased anthropometric indicators, which have been proposed in previous studies as the risk factors of cardiovascular diseases, increased visceral fat, irrespective of BMI and WHR, can also be a risk factor for the development of cardiovascular diseases.

In the present study, heart mass and heart mass to height ratio were higher in the case group than in the control group, which may have been due to higher epicardial fat as well as increased myocardial hypertrophy (caused by ischemia). These findings are consistent with the other results obtained in this study.

\subsection{Conclusions}

It should be noted that coronary artery obstruction is a possibility in cases that show an accumulation of epicardial fat in their echocardiograms, have an enlarged heart and show thick abdominal fat, especially around the navel, or an accumulation of mesenteric fat in their ultrasound or computed tomography scan, particularly in seemingly healthy men under 40 , even if their BMI and WHR are within the normal range.

\section{Acknowledgments}

Authors would like to thank their colleagues in legal medicine organization and Rajaie Cardiovascular medical and research center, particularly Dr. Hooman Bakhshandeh.

\section{References}

1. Canoy D. Coronary heart disease and body fat distribution. Curr Atheroscler Rep. 2010;12(2):125-33. doi: 10.1007/s11883-010-0092-9. [PubMed: 20425248]. 
2. Ashwell M. Obesity in men and women. Int J Obes Relat Metab Disord. 1994;18 Suppl 1:S1-7. [PubMed: 8087159].

3. Weinsier RL, Fuchs RJ, Kay TD, Triebwasser JH, Lancaster MC. Body fat: its relationship to coronary heart disease, blood pressure, lipids and other risk factors measured in a large male population. Am J Med. 1976;61(6):815-24. [PubMed: 1008068].

4. Zamboni M, Armellini F, Sheiban I, De Marchi M, Todesco T, BergamoAndreis IA, et al. Relation of body fat distribution in men and degree of coronary narrowings in coronary artery disease. Am J Cardiol. 1992;70(13):1135-8. [PubMed:1414934].

5. Kortelainen ML, Sarkioja T. Extent and composition of coronary lesions and degree of cardiac hypertrophy in relation to abdominal fatness in men under 40 years of age. Arterioscler Thromb Vasc Biol. 1997;17(3):574-9. [PubMed: 9102179].

6. Despres JP, Prud'homme D, Pouliot MC, Tremblay A, Bouchard C. Estimation of deep abdominal adipose-tissue accumulation from simple anthropometric measurements in men. Am J Clin Nutr. 1991;54(3):4717. [PubMed: 1877502].

7. Canoy D, Boekholdt SM, Wareham N, Luben R, Welch A, Bingham $S$, et al. Body fat distribution and risk of coronary heart disease in men and women in the European Prospective Investigation Into Cancer and Nutrition in Norfolk cohort: a population-based prospective study. Circulation. 2007;116(25):2933-43. doi: 10.1161/CIRCULATIONAHA.106.673756. [PubMed: 18071080$]$

8. Kortelainen ML, Sarkioja T. Visceral fat and coronary pathology in male adolescents. Int J Obes Relat Metab Disord. 2001;25(2):228-32. doi: 10.1038/sj.ijo.0801466. [PubMed: 11410824].

9. Kortelainen ML. Association between cardiac pathology and fat tissue distribution in an autopsy series of men without premortem evidence of cardiovascular disease. Int $J$ obes Relat metab disord. 1996;20(3):245-52.

10. Kortelainen ML, Sarkioja T. Coronary atherosclerosis and myocardial hypertrophy in relation to body fat distribution in healthy women: an autopsy study on 33 violent deaths. Int J Obes Relat Metab Disord. 1997;21(1):43-9. [PubMed: 9023600].

11. Rabkin SW. Epicardial fat: properties, function and relationship to obesity. Obes Rev. 2007;8(3):253-61. doi: 10.1111/j.1467789X.2006.00293.x. [PubMed: 17444966].

12. Hassan M, Latif N, Yacoub M. Adipose tissue: friend or foe?. Nat Rev Cardiol. 2012;9(12):689-702. doi: 10.1038/nrcardio.2012.148. [PubMed: 23149834].

13. Sacks HS, Fain JN. Human epicardial adipose tissue: a review. Am Heart J. 2007;153(6):907-17. doi: 10.1016/j.ahj.2007.03.019. [PubMed: 17540190 ]. 